\title{
Coupled Fixed Point Theorem for Weakly Compatible Mappings in Intuitionistic Fuzzy Metric Spaces
}

\author{
Sumitra \\ Department of Mathematics, S.R.Education, Institute , Haryana, India
}

\begin{abstract}
The aim of present paper is to introduce the notion of $t$ conorm of H-type analogous to t-norm of H-type introduced by Hadzic [9] and using this notion we prove coupled fixed point theorems for weakly compatible mappings in intuitionistic fuzzy metric spaces.
\end{abstract}

\section{Key Words}

Coupled fixed point, Compatible maps, Fixed points and Intuitionistic Fuzzy Metric Spaces.

\section{INTRODUCTION}

Recently, Bhaskar and Lakshmikantham [10] introduced the concepts of coupled fixed points and mixed monotone property and illustrated these results by proving the existence and uniqueness of the solution for a periodic boundary value problem. Later on these results were extended and generalized by Fang [4]and Xin-Qi Hu [12] etc .

As a generalization of fuzzy sets, Atanassove [1] introduced and studied the concept of intuitionistic fuzzy metric sets. Intuitionistic fuzzy sets deal with both degree of nearness and non-nearness. Motivated by the idea of intuitionistic fuzzy metric sets Park [8] introduced the concept of intuitionistic fuzzy metric spaces using continuous t-norms and continuous t-conorms. Later on , many authors[2-6] have studied fixed points results in intuitionistic fuzzy metric spaces.Fang [7] defined $\phi$-contractive conditions and proved some important fixed point theorems under $\phi$-contractions for compatible and weakly compatible maps in Menger PM-spaces using t-norm of H-type introduced by Hadzic [9].

In this paper, first we introduce the notion of t-conorm of $\mathrm{H}$ type analogous to t-norm of H-type introduced by Hadžíc [9] and using this notion we prove a common coupled fixed point theorem for compatible mappings in intuitionistic fuzzy metric spaces.

\section{DEFINITIONS}

AND PRELIMINARIES

For basic definitions and structure on intuitionistic fuzzy metric spaces we refer to [ 1-6 ].However we give some definitions in the sequel.

Definition 2.1 A binary operation $*:[0,1] \times[0,1] \rightarrow[0,1]$ is continuous t-norm if $0,1, *$ is a topological abelian monoid with unit 1 such that $\mathrm{t} a * \mathrm{~b} \leq \mathrm{c} * \mathrm{~d}$ whenever $\mathrm{a} \leq \mathrm{c}$ and $\mathrm{b} \leq \mathrm{d}$ for all $\mathrm{a}, \mathrm{b}, \mathrm{c}, \mathrm{d} \in[0,1]$.

Definition 2.2. A binary operation $\diamond:[0,1] \times[0,1] \rightarrow[0,1]$ is continuous t-conorm if $0,1, \diamond$ is a topological abelian monoid with unit 1 such that ) $\mathrm{a} \diamond \mathrm{b} \leq \mathrm{c} \diamond \mathrm{d}$ whenever $\mathrm{a} \leq \mathrm{c}$ and $\mathrm{b} \leq \mathrm{d}$ for all $\mathrm{a}, \mathrm{b}, \mathrm{c}, \mathrm{d} \in[0,1]$.

Definition 2.3[3]. A 5-tuple (X, M, N, *, $\diamond$ ) is said to be an intuitionistic fuzzy metric space if $\mathrm{X}$ is an arbitrary set, * is a continuous t-norm, $\diamond$ is a continuous t-conorm and $\mathrm{M}, \mathrm{N}$ are fuzzy sets on $X^{2} \times(0, \infty)$ satisfying the following conditions:

(i) $\mathrm{M}(\mathrm{x}, \mathrm{y}, \mathrm{t})+\mathrm{N}(\mathrm{x}, \mathrm{y}, \mathrm{t}) \leq 1$ for all $\mathrm{x}, \mathrm{y} \in \mathrm{X}$ and $\mathrm{t}>0$,

(ii) $\mathrm{M}(\mathrm{x}, \mathrm{y}, 0)=0$ for all $\mathrm{x}, \mathrm{y} \in \mathrm{X}$,

(iii) $\mathrm{M}(\mathrm{x}, \mathrm{y}, \mathrm{t})=1$ for all $\mathrm{x}, \mathrm{y} \in \mathrm{X}$ and $\mathrm{t}>0$ if and only if

$$
\mathrm{x}=\mathrm{y}
$$

(iv) $\mathrm{M}(\mathrm{x}, \mathrm{y}, \mathrm{t})=\mathrm{M}(\mathrm{y}, \mathrm{x}, \mathrm{t})$ for all $\mathrm{x}, \mathrm{y} \in \mathrm{X}$ and $\mathrm{t}>0$,

(v) $\mathrm{M}(\mathrm{x}, \mathrm{y}, \mathrm{t}) * \mathrm{M}(\mathrm{y}, \mathrm{z}, \mathrm{s}) \leq \mathrm{M}(\mathrm{x}, \mathrm{z}, \mathrm{t}+\mathrm{s})$ for all $\mathrm{x}, \mathrm{y}, \mathrm{z} \in \mathrm{X}$ and $\mathrm{t}, \mathrm{s}>0$

(vi) for all $\mathrm{x}, \mathrm{y} \in \mathrm{X}, \mathrm{M}(\mathrm{x}, \mathrm{y},):.[0, \infty) \rightarrow[0,1]$ is continuous,

(vii) $\lim _{t \rightarrow \infty} M(x, y, t)=1$, for all $\mathrm{x}, \mathrm{y}$ in $\mathrm{X}$,

(viii) $\mathrm{N}(\mathrm{x}, \mathrm{y}, 0)=1$ for all $\mathrm{x}, \mathrm{y} \in \mathrm{X}$,

(ix) $\mathrm{N}(\mathrm{x}, \mathrm{y}, \mathrm{t})=0$ for all $\mathrm{x}, \mathrm{y} \in \mathrm{X}$ and $\mathrm{t}>0$ if and only if

$x=y$,

(x) $N(x, y, t)=N(y, x, t)$ for all $x, y \in X$ and $t>0$,

(xi) $\mathrm{N}(\mathrm{x}, \mathrm{y}, \mathrm{t}) \diamond \mathrm{N}(\mathrm{y}, \mathrm{z}, \mathrm{s}) \geq \mathrm{N}(\mathrm{x}, \mathrm{z}, \mathrm{t}+\mathrm{s})$ for all $\mathrm{x}, \mathrm{y}, \mathrm{z} \in \mathrm{X}$ and $\mathrm{t}, \mathrm{s}>0$,

(xii) for all $\mathrm{x}, \mathrm{y} \in \mathrm{X}, \mathrm{N}(\mathrm{x}, \mathrm{y},):.[0, \infty) \rightarrow[0,1]$ is continuous,

(xiii) $\lim _{t \rightarrow \infty} N(x, y, t)=0$, for all $\mathrm{x}, \mathrm{y}$ in $\mathrm{X}$.

Then $(\mathrm{M}, \mathrm{N})$ is called an intuitionistic fuzzy metric on $X$. the functions $M(x, y, t)$ and $N(x, y, t)$ denote the degree of nearness and the degree of non-nearness between $\mathrm{x}$ and $\mathrm{y}$ with respect to $t$, respectively.

Definition 2.4[3]. Let $(X, M, N, *, \diamond)$ be an intuitionistic fuzzy metric space. Then

(i) a sequence $\left\{x_{n}\right\}$ in $\mathrm{X}$ is said to be a Cauchy sequence, if for all $\mathrm{t}>0$ and $\mathrm{p} \geq 1$, 
$\lim _{n \rightarrow \infty} M\left(x_{n+p}, x_{n}, t\right)=1, \lim _{n \rightarrow \infty} N\left(x_{n+p}, x_{n}, t\right)=0$.

(ii) a sequence $\left\{x_{n}\right\}$ in $\mathrm{X}$ is said to be convergent to a point

$\mathrm{x} \in \mathrm{X}$, if for all $\mathrm{t}>0$,

$\lim _{n \rightarrow \infty} M\left(x_{n}, x, t\right)=1, \lim _{n \rightarrow \infty} N\left(x_{n}, x, t\right)=0$.

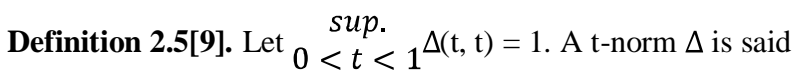
to be of H-type if the family of functions $\left\{\Delta^{m}(t)\right\}_{m=1}^{\infty}$ is equicontinuous at $\mathrm{t}=1$, where

$\Delta^{1}(t)=\mathrm{t}, \Delta^{m+1}(t)=\mathrm{t} \Delta\left(\Delta^{m}(t)\right), \mathrm{m}=1,2 \ldots \ldots \mathrm{t} \in[0,1]$.

The t-norm $\Delta_{M}=$ min. is an example of t-norm of H-type.

Remark 2.1. $\Delta$ is a H-type t-norm iff for any $\lambda \in(0,1)$, there exists $\delta(\lambda) \in(0,1)$ such that $\Delta^{m}(t)>(1-\lambda)$ for all $\mathrm{m} \in \mathrm{N}$, when $\mathrm{t}>(1-\delta)$.

We now define notion of t-conorm of H-type analogous to tnorm of $\mathrm{H}$-type as follows.

Definition 2.6. Let $\left.\begin{array}{c}\inf \text {. } \\ 0<t<1\end{array}\right\rangle(\mathrm{t}, \mathrm{t})=0$. A t-conorm $\Delta$ is said to be of H-type if the family of functions $\left\{\nabla^{m}(t)\right\}_{m=1}^{\infty}$ is equicontinuous at $\mathrm{t}=0$, where $\Delta^{1}(t)=\mathrm{t}, \nabla^{m+1}(t)=\mathrm{t} \Delta$ $\left(\nabla^{m}(t)\right), \mathrm{m}=1,2, \ldots \mathrm{t} \in[0,1]$.

The $\mathrm{t}$ - conorm $\diamond_{m}=$ max. is an example of $\mathrm{t}$-conorm of $\mathrm{H}$ type.

Remark 2.2. $\nabla$ is a H-type t-norm iff for any $\lambda \in(0,1)$, there exists $\delta(\lambda) \in(0,1)$ such that $\nabla^{m}(t)<\lambda$ for all $\mathrm{m} \in \mathrm{N}$, when

$\mathrm{t}<\delta$.

Definition 2.7[11]. An element ( $x, y) \in X \times X$ is called a coupled fixed point of the mapping $\mathrm{f}: \mathrm{X} \times \mathrm{X} \rightarrow \mathrm{X}$ if

$\mathrm{f}(\mathrm{x}, \mathrm{y})=\mathrm{x}, \quad \mathrm{f}(\mathrm{y}, \mathrm{x})=\mathrm{y}$.

Definition 2.8[11].An element $(\mathrm{x}, \mathrm{y}) \in \mathrm{X} \times \mathrm{X}$ is called a coupled coincidence point of the mappings $\mathrm{f}: \mathrm{X} \times \mathrm{X} \rightarrow \mathrm{X}$ and $\mathrm{g}: \mathrm{X} \rightarrow \mathrm{X}$ if

$f(x, y)=g(x), \quad f(y, x)=g(y)$.

Definition 2.9[11]. An element ( $x, y) \in X \times X$ is called

(i) a common coupled fixed point of the mappings $\mathrm{f}: \mathrm{X} \times \mathrm{X} \rightarrow \mathrm{X}$ and $\mathrm{g}: \mathrm{X} \rightarrow \mathrm{X}$ if

$$
\mathrm{x}=\mathrm{f}(\mathrm{x}, \mathrm{y})=\mathrm{g}(\mathrm{x}), \quad \mathrm{y}=\mathrm{f}(\mathrm{y}, \mathrm{x})=\mathrm{g}(\mathrm{y}) .
$$

(ii)

a common fixed point of the mappings

$$
\begin{gathered}
f: X \times X \rightarrow X \text { and } g: X \rightarrow X \text { if } \\
x=f(x, x)=g(x) .
\end{gathered}
$$

Definition 2.10 [5]. The mappings $\mathrm{f}: \mathrm{X} \times \mathrm{X} \rightarrow \mathrm{X}$ and $\mathrm{g}: \mathrm{X} \rightarrow$ $\mathrm{X}$ are said to be compatible if $\lim _{n \rightarrow \infty} M\left(g f\left(x_{n}, y_{n}\right), f\left(g\left(x_{n}\right), g\left(y_{n}\right)\right), t\right)=1$,

$\lim _{n \rightarrow \infty} M\left(g f\left(y_{n}, x_{n}\right), f\left(g\left(y_{n}\right), g\left(x_{n}\right)\right), t\right)=1$

And $\lim _{n \rightarrow \infty} N\left(g f\left(x_{n}, y_{n}\right), f\left(g\left(x_{n}\right), g\left(y_{n}\right)\right), t\right)=0$,

$\lim _{n \rightarrow \infty} N\left(g f\left(y_{n}, x_{n}\right), f\left(g\left(y_{n}\right), g\left(x_{n}\right)\right), t\right)=0$,

for all $\mathrm{t}>0$ whenever $\left\{\mathrm{x}_{\mathrm{n}}\right\}$ and $\left\{\mathrm{y}_{\mathrm{n}}\right\}$ are sequences in $\mathrm{X}$, such that

$$
\begin{gathered}
\lim _{n \rightarrow \infty} f\left(x_{n}, y_{n}\right)=\lim _{n \rightarrow \infty} g\left(x_{n}\right)=\mathrm{x}, \\
\lim _{n \rightarrow \infty} f\left(y_{n}, x_{n}\right)=\lim _{n \rightarrow \infty} g\left(y_{n}\right)=\mathrm{y},
\end{gathered}
$$

for some $\mathrm{x}, \mathrm{y}$ in $\mathrm{X}$.

Lemma 2.1[2]. Let (X, M, N, *, $)$ be an intuitionistic fuzzy metric space and for all $\mathrm{x}, \mathrm{y}$ in $\mathrm{X}, \mathrm{t}>0$, if there exists a number $\mathrm{k} \in(0,1)$ such that

$\mathrm{M}(\mathrm{x}, \mathrm{y}, \mathrm{kt}) \geq \mathrm{M}(\mathrm{x}, \mathrm{y}, \mathrm{t})$ and $\mathrm{N}(\mathrm{x}, \mathrm{y}, \mathrm{kt}) \leq \mathrm{N}(\mathrm{x}, \mathrm{y}, \mathrm{t})$ then $\mathrm{x}=\mathrm{y}$.

\section{MAIN RESULTS}

Theorem 3.1. Let $(X, M, N, *, \diamond)$ be a Complete Intuitionistic Fuzzy Metric Space, * being continuous t-norm of H-type and $\checkmark$ being continuous $\mathrm{t}-$ conorm of H-type. Let $\mathrm{A}, \mathrm{B}: \mathrm{X} \times \mathrm{X} \rightarrow$ $\mathrm{X}$ and $\mathrm{S}, \mathrm{T}: \mathrm{X} \rightarrow \mathrm{X}$ be four mappings satisfying the following conditions:

(3.1) $\mathrm{A}(\mathrm{X} \times \mathrm{X}) \subseteq \mathrm{T}(\mathrm{X}), \mathrm{B}(\mathrm{X} \times \mathrm{X}) \subseteq \mathrm{S}(\mathrm{X})$ and the pairs

$(\mathrm{A}, \mathrm{S})$ and $(\mathrm{B}, \mathrm{T})$ are weakly compatible

(3.2) $\mathrm{S}$ and $\mathrm{T}$ are continuous,

(3.3) $M(A(x, y), B(u, v), k t) \geq \phi[\operatorname{Min}\{M(S x, T u, t), M(A(x, y), S x, t), M(B(u, v), T u, t)\}]$

(3.4) $N(A(x, y), B(u, v), k t) \leq \psi[\operatorname{Max}\{N(S x, T u, t), N(A(x, y), S x, t)$,

$$
N(B(u, v), T u, t)\}]
$$

$\forall x, y, u, v \in X, k \in(0,1)$ where

$\phi, \psi: 0,1 \rightarrow 0,1$ are continuous functions such that

$\phi(t)>t, \phi(1)=1$ and $\psi(t)<t, \psi(0)=0$ for

$0<t<1$. Then A,B,S and Thave a unique common fixed point in $\mathrm{X}$, i.e there exists unique $\mathrm{x}$ in $\mathrm{X}$ such that $\mathrm{A}(\mathrm{x}, \mathrm{x})=\mathrm{T}(\mathrm{x})=\mathrm{B}(\mathrm{x}, \mathrm{x})=\mathrm{S}(\mathrm{x})=\mathrm{x}$

Proof: Let $\mathrm{x}_{0}, \mathrm{y}_{0}$ be two arbitrary points in $\mathrm{X}$. Since $\mathrm{A}(\mathrm{X} \times \mathrm{X})$ $\subseteq \mathrm{T}(\mathrm{X})$, we can choose $\mathrm{x}_{1}, \mathrm{y}_{1}$ in $\mathrm{X}$ such that $\mathrm{T}\left(\mathrm{x}_{1}\right)=\mathrm{A}\left(\mathrm{x}_{0}, \mathrm{y}_{0}\right)$, $\mathrm{T}\left(\mathrm{y}_{1}\right)=\mathrm{A}\left(\mathrm{y}_{0}, \mathrm{x}_{0}\right)$. Again, Since $\mathrm{B}(\mathrm{X} \times \mathrm{X}) \subseteq \mathrm{S}(\mathrm{X})$, we can choose $\mathrm{x}_{2}, \mathrm{y}_{2}$ in $\mathrm{X}$ such that $\mathrm{S}\left(\mathrm{x}_{2}\right)=\mathrm{B}\left(\mathrm{x}_{1}, \mathrm{y}_{1}\right)$ and 
$\mathrm{S}\left(\mathrm{y}_{2}\right)=\mathrm{B}\left(\mathrm{y}_{1}, \mathrm{x}_{1}\right)$. Continuing in this way, we can construct two sequences $\left\{z_{n}\right\}$ and $\left\{z_{n}^{\prime}\right\}$ in $\mathrm{X}$ such that

$z_{2 n+1}=\mathrm{A}\left(x_{2 n}, y_{2 n}\right)=\mathrm{T}\left(x_{2 n+1}\right)$

$z_{2 n+2}=\mathrm{B}\left(x_{2 n+1}, y_{2 n+1}\right)=\mathrm{S}\left(x_{2 n+2}\right)$

And,$z_{2 n+1}^{\prime}=\mathrm{A}\left(y_{2 n}, x_{2 n}\right)=\mathrm{T}\left(y_{2 n+1}\right)$

$z_{2 n+2}^{\prime}=\mathrm{B}\left(y_{2 n+1}, x_{2 n+1}\right)=\mathrm{S}\left(y_{2 n+2}\right)$, for all $\mathrm{n} \geq 0$.

Step 1: We first show that $\left\{z_{n}\right\}$ and $\left\{z_{n}^{\prime}\right\}$ are Cauchy sequences.Using (3.3) and (3.4),

$$
\begin{aligned}
& M\left(z_{2 n+1}, z_{2 n+2}, k t\right) \\
& =M\left(A\left(x_{2 n}, y_{2 n}\right), B\left(x_{2 n+1}, y_{2 n+1}\right), k t\right) \\
& \geq \phi\left[\operatorname{Min}\left\{\begin{array}{l}
M\left(S x_{2 n}, T x_{2 n+1}, t\right), \\
M\left(A\left(x_{2 n}, y_{2 n}\right), S x_{2 n}, t\right), \\
M\left(B\left(x_{2 n+1}, y_{2 n+1}\right), T x_{2 n+1}, t\right)
\end{array}\right\}\right] \\
& =\phi\left[\operatorname{Min}\left\{\begin{array}{l}
M\left(A\left(x_{2 n-1}, y_{2 n-1}\right), B\left(x_{2 n}, y_{2 n}\right), t\right), \\
M\left(A\left(x_{2 n}, y_{2 n}\right), B\left(x_{2 n-1}, y_{2 n-1}\right), t\right), \\
M\left(B\left(x_{2 n+1}, y_{2 n+1}\right), A\left(x_{2 n}, y_{2 n}\right), t\right)
\end{array}\right\}\right]
\end{aligned}
$$

$=\phi\left[\operatorname{Min}\left\{M\left(z_{2 n}, z_{2 n+1}, t\right)\right.\right.$,

$\left.\left.M\left(z_{2 n}, z_{2 n+1}, t\right), M\left(z_{2 n+2}, z_{2 n+1}, t\right)\right\}\right]$

If $M\left(z_{2 n+2}, z_{2 n+1}, t\right) \leq M\left(z_{2 n+1}, z_{2 n}, t\right)$, a

contradiction as $M(x, y, t)$ is increasing, therefore,

$$
\begin{aligned}
M\left(z_{2 n+2}, z_{2 n+1}, k t\right) & \geq \phi\left[M\left(z_{2 n+1}, z_{2 n}, t\right)\right] \\
& \geq M\left(z_{2 n+1}, z_{2 n}, t\right)
\end{aligned}
$$

And ,

$$
\left.\begin{array}{l}
N\left(z_{2 n+1}, z_{2 n+2}, k t\right) \\
=N\left(A\left(x_{2 n}, y_{2 n}\right), B\left(x_{2 n+1}, y_{2 n+1}\right), k t\right) \\
\leq \psi\left[\operatorname{Max}\left\{\begin{array}{l}
N\left(S x_{2 n}, T x_{2 n+1}, t\right), \\
N\left(A\left(x_{2 n}, y_{2 n}\right), S x_{2 n}, t\right), \\
N\left(B\left(x_{2 n+1}, y_{2 n+1}\right), T x_{2 n+1}, t\right)
\end{array}\right)\right]
\end{array}\right]
$$

$=\psi\left[\operatorname{Max}\left\{N\left(z_{2 n}, z_{2 n+1}, t\right), N\left(z_{2 n}, z_{2 n+1}, t\right)\right.\right.$,

$\left.\left.N\left(z_{2 n+2}, z_{2 n+1}, t\right)\right\}\right]$

If $N\left(z_{2 n+2}, z_{2 n+1}, t\right) \geq N\left(z_{2 n+1}, z_{2 n}, t\right)$, a

contradiction as $N(x, y, t)$ is decreasing, therefore,

$N\left(z_{2 n+2}, z_{2 n+1}, k t\right) \leq \psi\left[N\left(z_{2 n+1}, z_{2 n}, t\right)\right] \leq N\left(z_{2 n+1}, z_{2 n}, t\right)$

Similarly, we can show that

$$
\begin{aligned}
& M\left(z_{2 n+3}, z_{2 n+2}, k t\right) \geq M\left(z_{2 n+2}, z_{2 n+1}, t\right) \text { and } \\
& N\left(z_{2 n+3}, z_{2 n+2}, k t\right) \leq N\left(z_{2 n+2}, z_{2 n+1}, t\right)
\end{aligned}
$$

In general, $M\left(z_{n}, z_{n+1}, k t\right) \geq M\left(z_{n-1}, z_{n}, t\right)$ and $N\left(z_{n}, z_{n+1}, k t\right) \leq N\left(z_{n-1}, z_{n}, t\right)$. Thus by lemma [2.1] $z_{n}$ is a Cauchy sequence. Similarly we can show $\left\{z_{n}^{\prime}\right\}$ is Cauchy sequence.

Step 2: Since $\mathrm{X}$ is complete, there exists point a, b in $\mathrm{X}$ such that

$\lim _{n \rightarrow \infty} z_{n}=\mathrm{a}$ and $\lim _{n \rightarrow \infty} z_{n}^{\prime}=\mathrm{b}$,

that is, $\lim _{n \rightarrow \infty} z_{2 n+1}=\lim _{n \rightarrow \infty} \mathrm{A}\left(\mathrm{x}_{2 \mathrm{n}}, \mathrm{y}_{2 \mathrm{n}}\right)=\lim _{n \rightarrow \infty} \mathrm{T}\left(\mathrm{x}_{2 \mathrm{n}+1}\right)=\mathrm{a}$,

$\lim _{n \rightarrow \infty} z_{2 n}=\lim _{n \rightarrow \infty} \mathrm{B}\left(\mathrm{x}_{2 \mathrm{n}+1}, \mathrm{y}_{2 \mathrm{n}+1}\right)=\lim _{n \rightarrow \infty} \mathrm{S}\left(\mathrm{x}_{2 \mathrm{n}+2}\right)=\mathrm{a}$

and, $\lim _{n \rightarrow \infty} z_{2 n+1}^{\prime}=\lim _{n \rightarrow \infty} \mathrm{A}\left(\mathrm{y}_{2 \mathrm{n}}, \mathrm{x}_{2 \mathrm{n}}\right)=\lim _{n \rightarrow \infty} \mathrm{T}\left(\mathrm{y}_{2 \mathrm{n}+1}\right)=\mathrm{b}$,

$\lim _{n \rightarrow \infty} z_{2 n}^{\prime}=\lim _{n \rightarrow \infty} \mathrm{B}\left(\mathrm{y}_{2 \mathrm{n}+1}, \mathrm{x}_{2 \mathrm{n}+1}\right)=\lim _{n \rightarrow \infty} \mathrm{S}\left(\mathrm{y}_{2 \mathrm{n}+2}\right)=\mathrm{b}$.

We first show that $\mathrm{S}(\mathrm{a})=\mathrm{T}(\mathrm{a})$. As $\mathrm{S}$ and $\mathrm{T}$ are continuous , so $S S x_{2 n} \rightarrow S a, S S y_{2 n} \rightarrow S b, S A\left(x_{2 n}, y_{2 n}\right) \rightarrow S a$. and

$\operatorname{TTx}_{2 n} \rightarrow T a, T T y_{2 n} \rightarrow T b, T B\left(x_{2 n}, y_{2 n}\right) \rightarrow T a$.

But the pairs $(\mathrm{A}, \mathrm{S})$ and $(\mathrm{B}, \mathrm{T})$ are weakly compatible, so,

$S A\left(x_{2 n}, y_{2 n}\right)=A\left(S x_{2 n}, S y_{2 n}\right) \rightarrow S a$ and

$T B\left(x_{2 n}, y_{2 n}\right)=B\left(T x_{2 n}, T y_{2 n}\right) \rightarrow T a$.Using (3.3) and (3.4), we have,

$\left.M\left(A\left(S x_{2 n}, S y_{2 n}\right), B\left(T x_{2 n}, T y_{2 n}\right), k t\right)\right)$

$\geq \phi\left[\operatorname{Min}\left\{\begin{array}{l}M\left(S S x_{2 n}, T T x_{2 n}, t\right), \\ M\left(A\left(S x_{2 n}, S y_{2 n}\right), S S x_{2 n}, t\right), \\ M\left(B\left(T x_{2 n}, T y_{2 n}\right), T T x_{2 n}, t\right)\end{array}\right\}\right]$ 
Taking $n \rightarrow \infty$, we get

$M(\operatorname{Sa}, T a, k t) \geq \phi[\operatorname{Min}\{M(\operatorname{Sa}, T a, t), 1,1\}$

$$
\geq M(S a, T a, t)
$$

And ,

$\left.N\left(A\left(S x_{2 n}, S y_{2 n}\right), B\left(T x_{2 n}, T y_{2 n}\right), k t\right)\right)$

$\leq \psi\left[\operatorname{Max}\left\{\begin{array}{l}N\left(S S x_{2 n}, T T x_{2 n}, t\right), N\left(A\left(S x_{2}\right.\right. \\ N\left(B\left(T x_{2 n}, T y_{2 n}\right), T T x_{2 n}, t\right)\end{array}\right.\right.$

Taking $n \rightarrow \infty$, and using lemma (3.1), we get

$N(S a, T a, k t) \leq \psi[\operatorname{Min}\{N(S a, T a, t), 0,0\}]$

$$
\leq N(S a, T a, t) \text {. }
$$

Which gives $\mathrm{Sa}=\mathrm{Ta}$.

Now, we prove that $\mathrm{Sa}=\mathrm{B}(\mathrm{a}, \mathrm{b})$, again using (3.3) and (3.4) we have

$$
\begin{aligned}
& M\left(A\left(S x_{2 n}, S y_{2 n}\right), B(a, b), k t\right) \\
& \geq \phi\left[\operatorname { M i n } \left\{M\left(S S x_{2 n}, T a, t\right), M\left(A\left(S x_{2 n}, S y_{2 n}\right), S S x_{2 n}, t\right)\right.\right. \\
& M(B(a, b), T a, t)\}]
\end{aligned}
$$

And ,

$N\left(A\left(S x_{2 n}, S y_{2 n}\right), B(a, b), k t\right)$

$\leq \psi\left[\operatorname{Max}\left\{N\left(S S x_{2 n}, T a, t\right), N\left(A\left(S x_{2 n}, S y_{2 n}\right), S S x_{2 n}, t\right)\right.\right.$,

$N(B(a, b), T a, t)\}]$

Taking $n \rightarrow \infty$, we get $\mathrm{Sa}=\mathrm{B}(\mathrm{a}, \mathrm{b})=\mathrm{Ta}$. Now, we prove that $\mathrm{B}(\mathrm{a}, \mathrm{b})=\mathrm{A}(\mathrm{a}, \mathrm{b})$

$M(A(a, b), B(a, b), k t)$

$\geq \phi\left[\operatorname{Min}\left\{\begin{array}{l}M(S a, T a, t), M(A(a, b), S a, t), \\ M(B(a, b), T a, t)\end{array}\right\}\right.$

$=\phi[\operatorname{Min} 1, M(A(a, b), B(a, b), t), 1$

$>M(A(a, b), B(a, b), t)$

And ,

$N(A(a, b), B(a, b), k t)$

$\leq \psi\left[\operatorname{Max}\left\{\begin{array}{l}N(S a, T a, t), N(A(a, b), S a, t), \\ N(B(a, b), T a, t)\end{array}\right\}\right.$.

Thus, $\mathrm{A}(\mathrm{a}, \mathrm{b})=\mathrm{B}(\mathrm{a}, \mathrm{b})=\mathrm{Sa}=\mathrm{Ta}$. Similarly, we can show that $\mathrm{A}(\mathrm{b}, \mathrm{a})=\mathrm{B}(\mathrm{b}, \mathrm{a})=\mathrm{Sb}=\mathrm{Tb}$.

Let $\mathrm{A}(\mathrm{a}, \mathrm{b})=\mathrm{B}(\mathrm{a}, \mathrm{b})=\mathrm{Sa}=\mathrm{Ta}=\mathrm{x}$ and $\mathrm{A}(\mathrm{b}, \mathrm{a})=\mathrm{B}(\mathrm{b}, \mathrm{a})=$ $\mathrm{Sb}=\mathrm{Tb}=\mathrm{y}$. Since $(\mathrm{A}, \mathrm{S})$ and $(\mathrm{B}, \mathrm{T})$ are weakly compatible , so,

$$
\begin{aligned}
& S x=S A(a, b)=A(S a, S b)=A(x, y) \text { and } \\
& S y=S A(b, a)=A(S b, S a)=A(y, x) . \\
& T x=T B(a, b)=B(T a, T b)=B(x, y) \text { and } \\
& T y=T B(b, a)=B(T b, T a)=B(y, x) .
\end{aligned}
$$

Step 3: We next show that $x=y$. From (3.3) and (3.4),

$$
\begin{aligned}
& M(x, y, k t)=M(A(a, b), B(a, b), k t) \\
& \geq \phi\left[\operatorname{Min}\left\{\begin{array}{l}
M(S a, T a, t), M(A(a, b), S a, t) \\
M(B(a, b), T a, t)
\end{array}\right\}\right] \geq 1
\end{aligned}
$$

Therefore, $\mathrm{x}=\mathrm{y}$.

Step 4: Now, we prove that $\mathrm{Sx}=\mathrm{Tx}$, again using (3.3) and (3.4)

$M(S x, T x, k t)=M(A(x, y), B(x, y), k t)$

$\geq \phi\left[\operatorname{Min}\left\{\begin{array}{l}M(S x, T x, t), M(A(x, y), S x, t), \\ M(B(x, y), T x, t)\end{array}\right\}\right]$

$\geq M(S x, T x, t)$

$N(S x, T x, k t)=N(A(x, y), B(x, y), k t)$

$\leq \psi\left[\operatorname{Max}\left\{\begin{array}{l}N(S x, T x, t), N(A(x, y), S x, t), \\ N(B(x, y), T x, t)\end{array}\right\}\right]$

$\leq N(S x, T x, t)$

Thus we get $\mathrm{Sx}=\mathrm{Tx}$.

Step 5: Lastly, we prove that $S x=x$, From (3.3) and (3.4) , we have

$M(S x, x, k t)=M(A(x, y), B(x, y), k t)$

$\geq \phi\left[\operatorname{Min}\left\{\begin{array}{l}M(S x, T x, t), M(A(x, y), S x, t), \\ M(B(x, y), T x, t)\end{array}\right\} \geq 1\right.$

$N(S x, x, k t)=N(A(x, y), B(x, y), k t)$

$\leq \psi\left[\operatorname{Max}\left\{\begin{array}{l}N(S x, T x, t), N(A(x, y), S x, t), \\ N(B(x, y), T x, t)\end{array}\right\}\right] \leq 0$

Thus, we get $\mathrm{Sx}=\mathrm{x}$. Hence

$x=S x=T x=A(x, x)=B(x, x)$. This shows that $\mathrm{A}$, 
$\mathrm{B}, \mathrm{S}, \mathrm{T}$ have a common fixed point and uniqueness of $\mathrm{x}$ follows easily from (3.3) and (3.4).

Next we give an example in support of theorem 3.1

Example 3.1. Let $X=R$ and $d$ be the usual metric on $X$. Denote $a * b=a b$ and $a \diamond b=\min \{1, a+b\}$ for all $a, b$ in $[0,1]$ and let $\mathrm{M}_{\mathrm{d}}$ and $\mathrm{N}_{\mathrm{d}}$ be fuzzy sets on $\mathrm{X}^{2} \times(0, \infty)$ defined as follows:

$\mathrm{M}_{\mathrm{d}}(\mathrm{x}, \mathrm{y}, \mathrm{t})=\frac{t}{t+|x-y|}, \mathrm{N}_{\mathrm{d}}(\mathrm{x}, \mathrm{y}, \mathrm{t})=\frac{|x-y|}{t+|x-y|}$

Then $(\mathrm{X}, \mathrm{M}, \mathrm{N}, *, \diamond)$ is an intuitionistic fuzzy metric space.

Define the mappings

$A, B: X \times X \rightarrow X$ and $S, T: X \rightarrow X$ as follows

$A(x, y)=\left\{\begin{array}{l}x+y, x \in 0,2, y \in X \\ 1, \text { otherwise }\end{array}\right\}$ and

$B(x, y)=\left\{\begin{array}{l}x-y, x \in 0,2, y \in X \\ 2, \text { otherwise }\end{array}\right\}$

$S(x)=x$ and $T(x)=2 x$, then the pairs $(\mathrm{A}, \mathrm{S})$ and

$(\mathrm{B}, \mathrm{T})$ are weakly compatible and we see $\mathrm{A}(\mathrm{X} \times \mathrm{X}) \subseteq$

$\mathrm{T}(\mathrm{X}), \mathrm{B}(\mathrm{X} \times \mathrm{X}) \subseteq \mathrm{S}(\mathrm{X})$, So, all the conditions of our theorem are satisfied. Thus $\mathrm{A}, \mathrm{B}, \mathrm{S}$ and $\mathrm{T}$ have a unique common coupled fixed point in $\mathrm{X}$. Indeed, $\mathrm{x}=0$ is the unique common fixed point.

\section{REFERENCES}

[1] K. Atanassov. Intuitionistic fuzzy sets, Fuzzy Sets and Systems, vol. 20, pp. 87-96, 1986.

[2] C. Alaca, I. Altun and D. Turkoglu. On compatible mappings of type (I) and type (II) inintuitionistic fuzzy metric spaces, Commun. Korean Math. Soc., vol. 23, no. 3, pp. 427-446, 2008.

[3] C. Alaca, D. Turkoglu and C. Yildiz. Fixed points in intuitionistic fuzzy metric spaces, SolitionsChaos, Solitons\& Fractals, vol. 29, no. 5, pp. 1073-1078, 2006.

[4] D. Turkoglu, C. Alaca, Y. J. Cho, and C. Yildiz, Common fixed point theorems in intuitionistic fuzzy metric spaces, J. Appl. Math. \& Computing 22 (2006), 411-424.

[5] D. Turkoglu, C. Alaca, and C. Yildiz, Compatible mapsand compatible maps of types $(\alpha)$ and $(\beta)$ in intuitionistic fuzzy metric spaces, Demonstratio Math. 39 (2006), 671-684.

[6] D. Coker. An introduction to intuitionistic fuzzy topological spaces, Fuzzy Sets and System, vol.88, pp. 81-89, 1997.

[7] J.X.Fang, Common fixed point theorems of compatible and weakly compatible maps in Menger spaces, Nonlinear 